International Journal of Pure and Applied Mathematics

Volume 84 No. 5 2013, 613-632

ISSN: 1311-8080 (printed version); ISSN: 1314-3395 (on-line version)

url: http://www.ijpam.eu

doi: http://dx.doi.org/10.12732/ijpam.v84i5.13

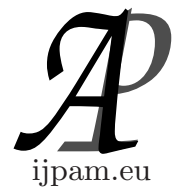

\title{
SHANNON'S INFORMATION THEORY AND ITS APPLICATIONS IN DERIVATIVE PRICING
}

\author{
Alexander Kushpel ${ }^{1 \S}$, Jeremy Levesley ${ }^{2}$ \\ Department of Mathematics \\ University of Leicester \\ Leicester, LE1 7RH, UK
}

\begin{abstract}
During the past two decades Lévy processes became very popular in Financial Mathematics. Truncated Lévy distributions were used for modeling by Mantegna and Stanley [13], [14]. Later Novikov [16] and Koponen [10] introduced a family of infinitely divisible analogs of these distributions. These models have been generalized by Boyarchenko and Levendorskii [5], and are known now as KoBoL models. Such models provide a good fit in many situations. The main aim of this article is to shed a fresh light onto the pricing theory using regular Lévy processes of exponential type. We introduce a class of payoff functions which is adopted to the set of regular Lévy processes of exponential type which is important in various applications. In particular, this class includes payoff function which corresponds to the European call option. We analyze pricing formula, construct and discuss several methods of approximation which are almost optimal in the sense of respective $n$-widths. This approach has its roots in Shannon's Information Theory.
\end{abstract}

AMS Subject Classification: 91G20, 30E10, 60G51, 91G60, 91G80.

Key Words: approximation, pricing theory, Lévy-driven models, $n$-width, information theory

\section{Introduction}

We shall study a common frictionless market consisting of a riskless bond and

Received: January 6, 2013

(C) 2013 Academic Publications, Ltd.

$\S$ Correspondence author url: www.acadpubl.eu 
stock which is modeled by an exponential Lévy process $S_{t}=e^{X_{t}}$ under a fixed equivalent martingale measure $\mathbb{Q}$ with a given constant riskless rate $r>0$. Since in our model the stock does not pay dividends then the discounted stock price $e^{-r t} S_{t}$ must be a martingale under $\mathbb{Q}$. Consider a contract (European call option) which gives to its owner the right but not the obligation to buy the underlying asset for the fixed strike price $K$ at the specified expiry date $T$. We need to evaluate its price $F_{\text {call }}\left(S_{t}, t\right)$. In this case the payoff has the form

$$
G(x)=\left(e^{x}-K\right)_{+},
$$

where for any $a \in \mathbb{R},(a)_{+}=\max \{a, 0\}, K$ is the strike price and $x=\ln \left(S_{t}\right)$. In the classical Merton-Black-Scholes (MBS) model the price of a stock follows the Geometric Brownian motion defined as $S_{t}=\exp \left(X_{t}\right)$, where $X_{t}, t \geq 0$ is the Brownian motion. It is well-known that MBS theory becomes much more efficient if additional stochastic factors are introduced. Consequently, it is important to consider a wider family of Lévy processes. Stable Lévy processes have been used first in this context by Mandelbrot [12] and Fama [9]. From the 90th Lévy processes became very popular (see, e.g., [13], [14], [4], [5] and references therein).

In this article there are several universal constants which enter into the estimates. These positive constants are mostly denoted by $C, C_{1}, \cdots$. For ease of notation we will write $a_{n} \asymp b_{n}$ if $C_{1} b_{n} \leq a_{n} \leq C_{2} b_{n}$ for any $n \in \mathbb{N}$.

\section{Characteristic Exponents and Density Functions}

For a finite measure $\mu$ on $\mathbb{R}$ we define its Fourier transform as

$$
\widehat{\mu}(y)=\mathbf{F} \mu(y)=\int_{\mathbb{R}} e^{-i x y} \mu(d x)
$$

and its formal inverse

$$
\mu(d x)=\mathbf{F}^{-1} \widehat{\mu}(d x)=\frac{1}{2 \pi} \int_{\mathbb{R}} e^{i x y} \widehat{\mu}(y) d y .
$$

It is known that if $\mu$ is infinitely divisible then there exists a unique continuous function $\phi: \mathbb{R} \rightarrow \mathbb{C}$ such that $\phi(0)=0$ and $e^{\phi(y)}=\widehat{\mu}(y)$. Hence, the characteristic function of the distribution of $X_{t}$ of any Lévy process can be represented in the form $\mathbb{E}\left[e^{i x X_{t}}\right]=e^{-t \psi(x)}$, where $x \in \mathbb{R}, t \in \mathbb{R}_{+}$and the function $\psi(x)$ is uniquely determined. This function is called the characteristic exponent. Vice 
versa, a Lévy process $X=\left\{X_{t}\right\}_{t \in \mathbb{R}_{+}}$is determined uniquely by its characteristic exponent $\psi(x)$. The key role in our analysis plays the following classical result known as the Lévy-Khintchine formula which gives a representation of characteristic functions of all infinitely divisible distributions.

Theorem 1. Let $X=\left\{X_{t}\right\}_{t \in \mathbb{R}_{+}}$be a Lévy process on $\mathbb{R}$. Then its characteristic exponent admits the representation

$$
\psi(y)=-\frac{1}{2} a y^{2}-i b y-\int_{\mathbb{R}}\left(1-e^{i y x}+i y x \chi_{[-1,1]}(x)\right) \Pi(d x),
$$

where $\chi_{[-1,1]}(x)$ is the characteristic function of $[-1,1], a \geq 0, b \in \mathbb{R}$ and $\Pi(x)$ is a measure on $\mathbb{R}$ such that

$$
\int_{\mathbb{R}} \min \left\{1, x^{2}\right\} \Pi(d x)<\infty, \Pi(\{0\})=0 .
$$

Hence $\widehat{\mu}(y)=e^{\psi(y)}$.

The density of $\Pi$ is known as the Lévy density. In particular, if $a=0$ then the Lévy process is a pure non-Gaussian process and if $\Pi=0$ the process is Gaussian. We say that the process is purely discontinuous if $a=b=0$ in (2). The systematic exposition of the theory of Lévy processes can be found in [6], [7], [8], [17], [2], [15].

It follows from (2) that for a purely discontinuous process $\psi(\xi)$ can be written as

$$
\psi(\xi)=\int_{\mathbb{R}}\left(1-e^{i \xi x}+i \xi x \chi_{[-1,1]}(x)\right) \Pi(d x) .
$$

We shall be concerned just with purely discontinuous processes. Applying (4) we get the following representation for the density function $p_{t}(y)$,

$$
\begin{gathered}
p_{t}(y)=\frac{1}{2 \pi} \int_{\mathbb{R}} e^{i y \xi} \mathbb{E}\left[e^{i \xi X_{t}}\right] d \xi=\mathbf{F}^{-1}\left(e^{-t \psi(\xi)}\right)(y)=\frac{1}{2 \pi} \int_{\mathbb{R}} e^{i y \xi-t \psi(\xi)} d \xi \\
=\frac{1}{2 \pi} \int_{\mathbb{R}} \exp \left(i y \xi-t \int_{\mathbb{R}}\left(1-e^{i \xi x}+i \xi x \chi_{[-1,1]}(x)\right) \Pi(d x)\right) d \xi .
\end{gathered}
$$

To find an explicit expression of the integral (5) we need to assume a very specific form of $\Pi(d x)$. Consequently, an explicit representations in this case is a real rarity. For instance, Barndorff and Nielsen [3] obtained the Lebesgue density function for generalized hyperbolic distributions which depends on five parameters

$$
d(x, \lambda, \alpha, \beta, \delta, \mu)=\frac{\left(\alpha^{2}-\beta^{2}\right)^{\lambda / 2}\left(\delta^{2}+(x-\mu)^{2}\right)^{(\lambda-1 / 2) / 2}}{(2 \pi)^{1 / 2} \alpha^{\lambda-1 / 2} \delta^{\lambda+1} K_{\lambda}\left(\alpha^{2}-\beta^{2}\right)}
$$




$$
\times K_{\lambda-1 / 2}\left(\alpha\left(\delta^{2}+2\left(x-\mu^{2}\right)\right)^{1 / 2}\right) e^{\beta(x-\mu)},
$$

where $K_{\sigma}(x)$ is the modified Bessel function of the third kind,

$$
K_{\sigma}(x)=\frac{1}{2} \int_{0}^{\infty} \xi^{\sigma-1} e^{-x\left(\xi-\xi^{-1}\right) / 2} d \xi .
$$

Motivated by the idea to consider a wider class of models we need to decide at which stage we should fall into numerical methods. If we still want to be strapped with an explicit form of the characteristic exponent but still agree to apply numerical methods to compute the density function $p_{t}(y)$ then we apply the following standard trick. Let $T$ be the expiry date, $t<T$ and $G\left(X_{T}\right)>0$ be the terminal payoff at the expiry date $T$, where $G(x)$ is defined by (1). The price of the European call option formally can be obtained from

$$
e^{-r t} F_{\text {call }}\left(S_{t}, t\right)=\mathbb{E}\left[e^{-r T} G\left(X_{T}\right) \mid X_{t}=x\right],
$$

where $e^{x}=S_{t}$ (see, e.g. [5], p. 104). It means that $F_{\text {call }}\left(S_{t}, t\right)$ can be formally written as

$$
F_{\text {call }}\left(S_{t}, t\right)=e^{-r \tau} \int_{\mathbb{R}} p_{\tau}(y-x) G(y) d y
$$

where $\tau:=T-t$ is the time to expiry. From the Lévy-Khintchine formula (4) it follows that it is possible to find $\psi(\xi)$ explicitly if we can compute explicitly the inverse Fourier transform of $\Pi(d x)$. Therefore, it was suggested by the authors of [5] to consider the following form of $\Pi(d x), \Pi(d x)=|x|^{\alpha} e^{-\beta|x|}$, where $\alpha$ and $\beta$ are fixed parameters. The following definitions are based on this observation. We say that a Lévy process is a regular Lévy process of exponential type if its density has at the origin a power type singularity and decays exponentially at the infinity. The characteristic exponent $\psi(\xi)$ of a regular Lévy process of exponential type admits an analytic extension onto the strip $\Im \xi \in\left(\lambda_{-}, \lambda_{+}\right)$, continuous on the boundary and admits the representation $\psi(\xi)=-i \mu \xi+\phi(\xi)$, where $\phi(\xi) \asymp|\xi|^{\nu}, \xi \rightarrow \infty$, $\xi \xi \in\left(\lambda_{-}, \lambda_{+}\right)$for some $\nu>0$. It is possible to show that for any regular Lévy process of exponential type the density function $p_{t}(y)$ is infinitely differentiable on $\mathbb{R}$ and exponentially decays with all of its derivatives as $y \rightarrow \pm \infty$.

Definition 2. Let $0<\nu \leq 2$ and $\lambda_{-}<0<\lambda_{+}$be some fixed parameters. Assume that

1. $\psi(\xi)$ admits the analytic extension into the strip $\lambda_{-}<\Im \xi<\lambda_{+}$;

2. $\psi^{0}(\xi):=\psi(\xi)+i \mu \xi$ is asymptotically positively homogeneous of order $\nu$ as $\xi \rightarrow \pm \infty$ in any closed strip $\omega_{-} \leq \Im \xi \leq \omega_{+}, \lambda_{-}<\omega_{-} \leq \omega_{+}<\lambda_{+}$. 
In this case we say that the corresponding Lévy process $X$ is a regular Lévy process of exponential type $\left(\lambda_{-}, \lambda_{+}\right)$and order $\nu$.

Let

$$
\Pi^{+}(\nu, \lambda, d x)=(\max \{x, 0\})^{-\nu-1} e^{-\lambda x} d x
$$

and

$$
\Pi^{-}(\nu, \lambda, d x)=(-\min \{0, x\})^{-\nu-1} e^{-\lambda x} d x,
$$

where $\nu<2$.

Definition 3. A Lévy process is called a KoBoL process of order $\nu<2$ if it is a purely discontinuous with the Lévy measure of the form $\Pi(d x)=$ $c_{+} \Pi^{+}\left(\nu, \lambda_{+}, d x\right)+c_{-} \Pi^{-}\left(\nu, \lambda_{-}, d x\right)$, where $c_{+}>0, c_{-}>0, \lambda_{-}<0<\lambda_{+}$.

We call $\nu$ the order of the process, $\lambda_{+}$and $\lambda_{-}$the steepness parameters and $c_{+}, c_{-}$the intensity parameters of the process. The parameter $\lambda_{-}\left(\lambda_{+}\right.$ respectively) determines the rate of the exponential decay of the right (left respectively) tail of the density function. It is easy to see that the condition (3) is satisfied, i.e.,

$$
\int_{\mathbb{R}} \min \left\{1, x^{2}\right\}\left(c_{+} \Pi^{+}\left(\nu, \lambda_{+}, d x\right)+c_{-} \Pi^{-}\left(\nu, \lambda_{-}, d x\right)\right)<\infty .
$$

It is known that if $\nu<2, \nu \neq 0,1$, then

$$
\begin{gathered}
\psi(\xi)=-i \mu \xi+c_{+} \Gamma(-\nu)\left[\left(-\lambda_{-}\right)^{\nu}-\left(-\lambda_{-}-i \xi\right)^{\nu}\right] \\
+c_{-} \Gamma(-\nu)\left[\lambda_{+}^{\nu}-\left(\lambda_{+}+i \xi\right)^{\nu}\right] .
\end{gathered}
$$

If $\nu=0$, then

$$
\begin{gathered}
\psi(\xi)=-i \mu \xi+c_{+}\left[\ln \left(-\lambda_{-}-i \xi\right)-\ln \left(-\lambda_{-}\right)\right] \\
+c_{-}\left[\ln \left(\lambda_{+}+i \xi\right)-\ln \lambda_{+}\right] .
\end{gathered}
$$

If $\nu=1$, then

$$
\begin{gathered}
\psi(\xi)=-i \mu \xi+c_{+}\left[\left(-\lambda_{-}\right) \ln \left(-\lambda_{-}\right)-\left(-\lambda_{-}-i \xi\right) \ln \left(-\lambda_{-}-i \xi\right)\right] \\
+c_{-}\left[\lambda_{+} \ln \lambda_{+}-\left(\lambda_{+}+i \xi\right) \ln \left(\lambda_{+}+i \xi\right)\right]
\end{gathered}
$$

where $\mu \in \mathbb{R}, c_{ \pm}>0$, and $\lambda_{-}<0<\lambda_{+}$(see [5], p.70). It is easy to see that the triple $\left(\nu, c_{+}, c_{-}\right)$determines the probability density. For larger $\nu$ and $c_{ \pm}$we get a larger peak of the probability distribution. The parameters $c_{+}$and $c_{-}$control asymmetry of the probability distribution while $\lambda_{-}$and $\lambda_{+}$determine the rate of exponential decay as $\xi \rightarrow \pm \infty$. 


\section{Optimal Bounds}

Our main aim is to approximate the function $F_{\text {call }}$ defined by (6). Remark that in [11] to approximate the integral (6) the authors used a piecewise linear approximation on a uniform mesh with step $h>0$. Observe that such kind of approximation has saturation of order $O\left(h^{2}\right)$ and can not reflect analytic properties of the characteristic exponent $\psi(\xi)$. Possible use of polynomial splines of higher degree, $r \geq 2$ significantly increase complexity of computations and gives the order of saturation $O\left(h^{r+1}\right)$ which is also too far from the optimal rate of convergence. The same argument is applicable to the possible wavelet approximations. Since any sequence of algebraic polynomials is not uniformly convergent on $\mathbb{R}$ (except of stationary sequences) then any continuous function which is not a polynomial can not be uniformly approximated. If we try to use rational approximations then we will be able to approximate just such continuous functions $f(x)$ that $\lim _{x \rightarrow \infty} f(x)=c$, where $c \in \mathbb{R}$ is some constant, which is not sufficient for our applications.

To compare and determine which apparatus of approximation is better over a wide range of methods of approximation we will need to set up respective extremal problem. The key problem here is that the information regarding smoothness of characteristic exponents $\psi(\xi)$ which are important in practical applications is given implicitly. This fact creates a range of significant difficulties of a fundamental nature. Of course, in such settings an analytic structure of the best possible algorithm would be too complex to be useful in applications. In the periodic case the most natural (and in many important cases optimal in the sense of the respective $n$-widths) method to approximate (6) is to use trigonometric approximations. In the case of approximation on the whole real line $\mathbb{R}$ the role of subspaces of trigonometric polynomials play functions from the Wiener spaces $W_{\sigma}(\mathbb{R})$, i.e., entire functions from $L_{2}(\mathbb{R})$ whose Fourier transform has support $[-\sigma, \sigma]$. Such functions have an exponential type $\sigma>0$. Remind that a function $f(z)$ defined on the complex plane $\mathbb{C}$ is of exponential type $\sigma>0$ if there exists a constant $M$ such that $|f(z)| \leq M e^{\sigma r}, z=r e^{i \theta}$ for any $\theta \in[0,2 \pi)$ in the limit of $r \rightarrow \infty$. Remark that entire functions of exponential type $\sigma$ as an apparatus of approximation was first considered by Bernstein [1].

To compare different methods of approximation we need to introduce Kolmogorov's $n$-width of a symmetric with respect to the origin set $A$ in a Banach space $X$. To be able to consider $n$-width of function classes defined on locally compact Abelian groups we use the notion of average dimension introduced by Tikhomirov [21]. 
Let $\mathbb{M}$ be a homogeneous locally compact space on which a group $\mathbb{G}$ acts. Assume that $\mathbb{M}$ is equipped with the invariant measure $d \nu$ and metric $d(\cdot, \cdot)$ with respect to $\mathbb{G}$. For any fixed $\mathbf{x} \in \mathbb{M}$ define $\mathbb{M}_{\eta}=\mathbb{M}_{\eta}(\mathbf{x}):=\{\mathbf{z} \in \mathbb{M} \mid d(\mathbf{x}, \mathbf{z}) \leq \eta\}$. Consider the collection of all subspaces $L \subset L_{p}(\mathbb{M})$ such that the operator $P_{\eta}$ of restricting,

$$
\begin{array}{ccc}
P_{\eta}: L & \longrightarrow & \mathbb{M}_{\eta}(\mathbf{x}) \cap L_{p}(\mathbb{M}) \\
f & \longmapsto & P_{\eta} f
\end{array}
$$

is compact and the quantity

$$
K_{\epsilon}\left(\eta, L, L_{p}(\mathbb{M})\right):=\min \left\{n \in \mathbb{Z}_{+} \mid d_{n}\left(L \cap U_{p}\left(\mathbb{M}_{\eta}\right), L_{p}\left(\mathbb{M}_{\eta}\right)\right)<\epsilon\right\},
$$

where $U_{p}(\mathbb{M}):=\left\{g \mid\|g\|_{p} \leq 1\right\}$. We put $K_{\epsilon}\left(\eta, L, L_{p}(\mathbb{M})\right)=+\infty$ if

$$
\left\{n \in \mathbb{Z}_{+} \mid d_{n}\left(L \cap U_{p}\left(\mathbb{M}_{\eta}\right), L_{p}\left(\mathbb{M}_{\eta}\right)\right)<\epsilon\right\}=\varnothing .
$$

It is easy to check that the function $\eta \rightarrow K_{\epsilon}\left(\eta, L, L_{p}(\mathbb{M})\right)$ is non-decreasing for any $\epsilon>0$ and $\epsilon \rightarrow K_{\epsilon}\left(\eta, L, L_{p}(\mathbb{M})\right)$ is non-increasing for any $\eta>0$.

Definition 4. Let $\varphi: \mathbb{R}_{+} \rightarrow \mathbb{R}_{+}$be a non-decreasing function. The quantity

$$
\operatorname{dim}\left(L, L_{p}(\mathbb{M}), \varphi\right):=\lim _{\epsilon \rightarrow 0} \lim _{\eta \rightarrow \infty} \frac{K_{\epsilon}\left(\eta, L, L_{p}(\mathbb{M})\right)}{\varphi(\eta)}
$$

is called the $\varphi$-average dimension of $L$ in $L_{p}(\mathbb{M})$. In particular, if $\mathbb{M}=\mathbb{R}$ and $\varphi(\eta)=2 \eta$, then the $\varphi$-average dimension is called the average dimension and is denoted by $\overline{\operatorname{dim}}\left(L, L_{p}(\mathbb{R})\right)$.

The idea of this general definition is based on

Theorem 5. (Whitteker-Kotel'nicov-Shannon) Let $f \in W_{\sigma}$ then

$$
f(x)=\sum_{k \in \mathbb{Z}} f\left(\frac{\pi k}{\sigma}\right) \frac{\sin (\sigma(x-\pi k / \sigma))}{\sigma(x-\pi k / \sigma)} .
$$

It means that any function $f \in W_{\sigma}$ can be reconstructed from its values on the set of points $\{\pi k / \sigma\}_{k \in \mathbb{Z}}$. It is shown in [21], p.367 that

$$
\overline{\operatorname{dim}}\left(W_{\sigma}, L_{2}(\mathbb{R})\right)=\frac{\sigma}{\pi}
$$

which is the inverse of the distance between the mesh points $\{\pi k / \sigma\}_{k \in \mathbb{Z}}$. Similarly, to recover any trigonometric polynomial

$$
t_{n}(x) \in \mathcal{T}_{n}:=\operatorname{lin}\{1, \cos k x, \sin k x, 1 \leq k \leq n\}
$$


we need to know its values at $2 n+1$ points $\{2 \pi k /(2 n+1), 1 \leq k \leq 2 n+1\}$. Remark that $\operatorname{dim} \mathcal{T}_{n}=2 n+1$.

Observe that Shannon [18]-[20] was the first who introduced the notion of average dimension in order to compare massivity of sets. His idea is based on the notion of entropy of a random object. Later, Kolmogorov defined an average dimension in terms of $\epsilon$-entropy of a set of non-random functions. This line of research has been developed by Tikhomirov [21] and others.

Definition 6. Let $A$ be centrally symmetric set, $A \subset L_{p}(\mathbb{M})$. The average Kolmogorov $\nu$-width is

$$
\bar{d}_{\nu}\left(A, L_{p}(\mathbb{M})\right):=\inf \sup _{f \in A} \inf _{g \in L}\|f-g\|_{L_{p}(\mathbb{M})},
$$

where inf is taken over all subspaces of dimension $\nu>0$.

Let $f$ be a measurable function on $\mathbb{R}$. By $f^{+}$we denote the non-increasing rearrangement of $f$, i.e., $f^{+}(t):=\inf \{\alpha>0 \mid \lambda(\alpha) \leq t, 0<t<\infty\}$, where $\lambda(\alpha):=$ meas $\{x \in \mathbb{R} \mid f(x) \geq \alpha\}$. Let $\mathcal{J}$ be the set of Jordan measurable functions on $\mathbb{R}$. It is known [21] that

Theorem 7. Let $K \in L_{2}(\mathbb{R}), \mathbf{F} K \in \mathcal{J}(\mathbb{R}), \rho>0$. Then

$$
\overline{d_{\rho}}\left(K * U_{2}(\mathbb{R}), L_{2}(\mathbb{R})\right)=(\mathbf{F} K(\rho))^{+} .
$$

Definition 8. We say that a regular Lévy process of exponential type $\left(\lambda_{-}, \lambda_{+}\right)$is adopted to the payoff function $G(x), G(x)=0, x \leq 0$ if for some $\omega<\lambda_{+}$we have $G(x) \leq C e^{\omega x}$ for any $x \geq 0$ and some absolute constant $C>0$. We denote by $\mathcal{G}\left(\lambda_{+}\right)$such class of payoff functions.

Theorem 9. Let $X_{t}$ be a regular Lévy process of exponential type $\left(\lambda_{-}, \lambda_{+}\right)$ and order $\nu$ adopted to the class of payoff functions $\mathcal{G}\left(\lambda_{+}\right)$. Then

$$
\overline{d_{\rho}}\left(p_{t} * \mathcal{G}\left(\lambda_{+}\right), L_{2}(\mathbb{R})\right) \leq C_{1} e^{-t \mu \omega} e^{-C_{2} t|\rho|^{\nu}}, \rho \rightarrow \infty .
$$

In particular, let $X_{t}$ be a $\mathrm{KoBoL}$ process of order $0<\nu<2$ with the intensity parameter $c=c_{+}=c_{-}$. Then

$$
\overline{d_{\rho}}\left(p_{t} * \mathcal{G}\left(\lambda_{+}\right), L_{2}(\mathbb{R})\right) \leq C_{3}\left\{\begin{array}{cc}
e^{c t \Gamma(-\nu) \cos (\nu \pi / 2) \rho^{\nu}}, & 0<\nu<2, \nu \neq 0,1, \\
e^{-c t \rho}, & \nu=1, \\
\rho^{-2 c t}, & \nu=0,
\end{array}\right.
$$

where $C_{1}, C_{2}$ and $C_{3}$ are some positive absolute constants. 
Proof. Consider a regular Lévy process of exponential type $\left(\lambda_{-}, \lambda_{+}\right)$which is adopted to the class of payoff functions $\mathcal{G}\left(\lambda_{+}\right)$. For a fixed $R>0$ define the contour

$$
\Gamma=[-R, R] \cup[R, i \omega+R] \cup[i \omega+R, i \omega-R] \cup[i \omega-R,-R] .
$$

Since $\psi(\xi)$ is analytic in the strip $\Im \xi \in\left(\lambda_{-}, \lambda_{+}\right)$then applying Cauchy's theorem we get

$$
\oint_{\Gamma} e^{i y \xi-t \psi(\xi)} d \xi=0
$$

Hence, since $\psi(\xi)$ is a characteristic exponent of a regular Lévy process of exponential type $\left(\lambda_{-}, \lambda_{+}\right)$then for some $\mu \in \mathbb{R}$ we have $\psi(\xi)=-i \mu \xi+\phi(\xi)$, where $\phi(\xi) \asymp|\xi|^{\nu}$ as $\xi \rightarrow \infty$ in $\Im \xi \in\left(\lambda_{-}, \lambda_{+}\right)$. It means that for any $t>0$,

$$
\lim _{R \rightarrow \infty}\left|\left(\int_{[R, i \omega+R]}+\int_{[i \omega-R,-R]}\right) e^{i y \xi-t \psi(\xi)} d \xi\right| \leq 2 \omega \lim _{R \rightarrow \infty} \exp \left(-t|R|^{\nu}\right)=0
$$

and

$$
\begin{gathered}
p_{t}(y)=\frac{1}{2 \pi} \int_{\mathbb{R}} e^{i y \xi-t \psi(\xi)} d \xi=\frac{1}{2 \pi} \lim _{R \rightarrow \infty} \int_{[-R, R]} e^{i y \xi-t \psi(\xi)} d \xi \\
=\frac{1}{2 \pi} \lim _{R \rightarrow \infty}\left(\int_{[R, i \omega+R]}+\int_{[i \omega-R, i \omega+R]}+\int_{[i \omega-R,-R]}\right) e^{i y \xi-t \psi(\xi)} d \xi \\
=\frac{1}{2 \pi} \lim _{R \rightarrow \infty} \int_{[i \omega-R, i \omega+R]} e^{i x \xi-t \psi(\xi)} d \xi=\frac{1}{2 \pi} \int_{[i \omega-\infty, i \omega+\infty]} e^{i y \xi-t \psi(\xi)} d \xi .
\end{gathered}
$$

Changing variable in the last integral, $z=\xi-i \omega$ we get

$$
p_{t}(y)=\frac{1}{2 \pi} \int_{\mathbb{R}} e^{i y(i \omega+z)-t \psi(i \omega+z)} d z=\frac{1}{2 \pi} e^{-\omega y} \int_{\mathbb{R}} e^{i y z-t \psi(i \omega+z)} d z .
$$

Since a regular Lévy process of exponential type $\left(\lambda_{-}, \lambda_{+}\right)$is adopted to the class $\mathcal{G}\left(\lambda_{+}\right)$then for some absolute constant $C>0$ we have $e^{-\omega y} \mathcal{G}\left(\lambda_{+}\right) \subset C U_{2}(\mathbb{R})$. Observe that

$$
K(y):=\frac{1}{2 \pi} \int_{\mathbb{R}} e^{i y z-t \psi(i \omega+z)} d z=\mathbf{F}^{-1}\left(e^{-t \psi(i \omega+\cdot)}\right)(y)
$$

and by the Paley-Wiener theorem we get

$$
\mathbf{F} K(\rho)=\mathbf{F} \circ \mathbf{F}^{-1}\left(e^{-t \psi(i \omega+\cdot)}\right)(\rho)=e^{-t \psi(i \omega+\rho)} .
$$


For any regular Lévy process of exponential type $\psi(\xi)=-i \mu \xi+\phi(\xi)$, where $\phi(\xi) \asymp|\xi|^{\nu}$ as $\xi \rightarrow \infty$ in $\Im \xi \in\left(\lambda_{-}, \lambda_{+}\right)$. Hence for some absolute constant $C>0$,

$$
\begin{gathered}
|\mathbf{F} K(\rho)|=|\exp (-t \psi(i \omega+\rho))|=|\exp (-t(-i \mu(i \omega+\rho)+\phi(i \omega+\rho)))| \\
|\mathbf{F} K(\rho)|=e^{-t \mu \omega}|\exp (-t \phi(i \omega+\rho))| \asymp e^{-t \mu \omega} e^{-C t|\rho|^{\nu}}, \rho \rightarrow \infty .
\end{gathered}
$$

Consequently, $(\mathbf{F} K(\rho))^{+} \asymp e^{-t \mu \omega} e^{-C t|\rho|^{\nu}}, \rho \rightarrow \infty$ and by Theorem 7

$$
\overline{d_{\rho}}\left(p_{t} * U_{2}(\mathbb{R}), L_{2}(\mathbb{R})\right) \asymp e^{-t \mu \omega} e^{-C t|\rho|^{\nu}}, \rho \rightarrow \infty .
$$

In particular,

$$
\overline{d_{\rho}}\left(p_{t} * \mathcal{G}\left(\lambda_{+}\right), L_{2}(\mathbb{R})\right) \leq C_{1} e^{-t \mu \omega} e^{-C_{2} t|\rho|^{\nu}}, \rho \rightarrow \infty .
$$

Consider asymptotic behavior of $\mathrm{KoBoL}$ exponent $\psi(\xi)$ in the strip $\Im \xi \in$ $\left(\lambda_{-}, \lambda_{+}\right),|\xi| \rightarrow \infty$. Direct calculation shows

$$
\lim _{\Im \xi \in\left(\lambda_{-}, \lambda_{+}\right),|\xi| \rightarrow \infty} \frac{\psi(\xi)}{\eta(\Re \xi)}=1,
$$

where

$\eta(x):=\left\{\begin{array}{cc}-i \mu x-\Gamma(-\nu) x^{\nu}\left(c_{+} e^{-i \nu \pi / 2}+c_{-} e^{i \nu \pi / 2}\right), & 0<\nu<2, \nu \neq 0,1, \\ -i\left(\mu x+c_{+} x \ln x-c_{-} x \ln x\right)+\left(c_{+}+c_{-}\right) x \pi / 2, & \nu=1, \\ -i \mu x+\left(c_{+}+c_{-}\right) \ln x, & \nu=0 .\end{array}\right.$

In particular, if $c_{+}=c_{-}=c$ then

$$
\eta(x):=\left\{\begin{array}{cc}
-i \mu x-\Gamma(-\nu) x^{\nu} \cos (\nu \pi / 2), & 0<\nu<2, \nu \neq 0,1 \\
-i \mu x+\pi c x, & \nu=1 \\
-i \mu x+2 c \ln x, & \nu=0 .
\end{array}\right.
$$

Applying Theorem 7 we get

$$
\begin{gathered}
\overline{d_{\rho}}\left(p_{t} * \mathcal{G}\left(\lambda_{+}\right), L_{2}(\mathbb{R})\right) \leq \overline{d_{\rho}}\left(p_{t} * U_{2}(\mathbb{R}), L_{2}(\mathbb{R})\right) \\
\asymp\left\{\begin{array}{cc}
e^{c t \Gamma(-\nu) \cos (\nu \pi / 2) \rho^{\nu}}, & 0<\nu<2, \nu \neq 0,1, \\
e^{-c t \rho}, & \nu=1, \\
\rho^{-2 c t}, & \nu=0 .
\end{array}\right.
\end{gathered}
$$




\section{Upper Bounds}

Denote by $W_{a}(\mathbb{R})$ the space of functions $f \in L_{2}(\mathbb{R})$ such that $\operatorname{supp} \mathbf{F} f \subset$ $[-a, a]$. The next result which is a generalisation of a well-known WhitekkerKotel'nikov-Shannon theorem has its roots in the Information Theory first developed by Shannon [18], [19], [20]. We construct a family of linear operators $\mathbf{P}_{\lambda_{a}}, a>0$,

$$
\begin{array}{ccc}
\mathbf{P}_{\lambda_{a}}: C(\mathbb{C}) & \longrightarrow & W_{2 a}(\mathbb{R}) \\
f(z) & \longmapsto & \left(\mathbf{P}_{\lambda_{a}} f\right)(a)
\end{array}
$$

such that for some absolute constant $C>0$ and any $a>0$ we have

$$
\left\|\mathbf{P}_{\lambda_{a}} \mid C(\mathbb{C}) \longrightarrow C(\mathbb{C})\right\|<C
$$

and $\left(\mathbf{P}_{\lambda_{a}} f\right)(z)=f(z)$ for any $f(z) \in W_{a}(\mathbb{R})$.

Theorem 10. Let $\lambda_{a}: \mathbb{R} \longrightarrow \mathbb{R}$ be any continuous function such that $\lambda_{a}(y)=1$ if $y \in[-a, a], \lambda_{a}(y)=0$ if $y \in \mathbb{R} \backslash[-2 a, 2 a]$ and

$$
J_{m, \lambda_{a}}(x)=\frac{1}{2 a}\left(\mathbf{F} \lambda_{a}\right)\left(-x+\frac{\pi m}{a}\right) .
$$

Then

$$
f(x)=\sum_{m \in \mathbb{Z}} f\left(\frac{\pi m}{a}\right) J_{m, \lambda_{a}}(x)
$$

for any $f \in W_{a}(\mathbb{R})$. In particular, if $\lambda_{a}^{*}:=\lambda^{*}(x / a), a>0$, where

$$
\lambda^{*}(x):=\left\{\begin{array}{cc}
0, & x \leq-1, \\
2 x+2, & -1 \leq x \leq-1 / 2 \\
1, & -1 / / 2 \leq x<1 / 2 \\
-2 x+2 & 1 / 2 \leq x<1 \\
0, & x \geq 1
\end{array}\right.
$$

then $\left\|\mathbf{P}_{\lambda_{a}^{*}} \mid C(\mathbb{C}) \longrightarrow C(\mathbb{C})\right\| \leq 3.5, \forall a>0$.

Proof. For any $f \in W_{a}(\mathbb{R})$ we have

$$
f(x)=\frac{1}{2 \pi} \int_{\mathbb{R}}(\mathbf{F} f)(y) e^{i y x} d y=\frac{1}{2 \pi} \int_{[-a, a]} \lambda_{a}(y)(\mathbf{F} f)(y) e^{i y x} d y
$$

because $\operatorname{supp} \mathbf{F} f \subset[-a, a]$. Since the set

$$
\varrho_{m}(y, a):=(2 a)^{-1 / 2} \exp \left(\frac{i \pi m y}{a}\right), m \in \mathbb{Z}
$$


is an orthonormal basis in $[-a, a]$ then $(\mathbf{F} f)(y)$ can be represented in $L_{2}(\mathbb{R})$ as

$$
(\mathbf{F} f)(y)=\sum_{m \in \mathbb{Z}} \alpha_{m} \varrho_{m}(y, a) .
$$

Using Plancherel's theorem we find that

$$
\begin{aligned}
& \alpha_{m}=\int_{[-a, a]}(\mathbf{F} f)(y) \varrho_{m}(-y, a) d y=\int_{\mathbb{R}}(\mathbf{F} f)(y) \varrho_{m}(-y, a) d y \\
& =2 \pi(2 a)^{-1 / 2}\left(\mathbf{F}^{-1} \circ \mathbf{F} f\right)\left(-\frac{\pi m}{a}\right)=2 \pi(2 a)^{-1 / 2} f\left(-\frac{\pi m}{a}\right) .
\end{aligned}
$$

Since $\operatorname{supp} \lambda_{a} \subset[-2 a, 2 a]$ then applying Plancherel's theorem again we get

$$
\begin{aligned}
& f(x)=\frac{1}{2 \pi} \int_{\mathbb{R}} \sum_{m \in \mathbb{Z}} 2 \pi(2 a)^{-1 / 2} f\left(-\frac{\pi m}{a}\right) \lambda_{a}(y) \varrho_{m}(y, a) e^{i x y} d y \\
& =\frac{1}{2 \pi} \int_{[-2 a, 2 a]} \sum_{m \in \mathbb{Z}} 2 \pi(2 a)^{-1 / 2} f\left(-\frac{\pi m}{a}\right) \lambda_{a}(y) \varrho_{m}(y, a) e^{i x y} d y \\
& =(2 a)^{-1} \sum_{m \in \mathbb{Z}} f\left(-\frac{\pi m}{a}\right) \int_{[-2 a, 2 a]} \lambda_{a}(y) \exp \left(i x y+\frac{i \pi m y}{a}\right) d y
\end{aligned}
$$

Changing the index of summation and simplifying we get

$$
f(x)=\sum_{m \in \mathbb{Z}} f\left(\frac{\pi m}{a}\right) J_{m, \lambda_{a}}(x),
$$

where

$$
\begin{gathered}
J_{m, \lambda_{a}}(x)=(2 a)^{-1} \int_{[-2 a, 2 a]} \lambda_{a}(y) \exp \left(i\left(x+\frac{i \pi m}{a}\right) y\right) d y \\
=(2 a)^{-1}\left(\mathbf{F} \lambda_{a}\right)\left(-x-\frac{\pi m}{a}\right) .
\end{gathered}
$$

In particular, let $\lambda_{a}=\lambda_{a}^{*}$. Direct calculation shows that

$$
\mathbf{F} \lambda_{a}^{*}(x)=a \mathbf{F} \lambda_{1}^{*}(a x)=4 a x^{-2}(\cos (a x / 2)-\cos a x) .
$$

Hence, for the norm of $\mathbf{P}_{\lambda_{a}^{*}}$ we have the following estimate

$$
\left\|\mathbf{P}_{\lambda_{a}^{*}} \mid C(\mathbb{C}) \longrightarrow C(\mathbb{C})\right\|:=\sup _{|f| \leq 1}\left\|\mathbf{P}_{\lambda_{a}^{*}} f\right\|
$$




$$
\begin{array}{r}
=\sup _{|f| \leq 1}\left|\sum_{m \in \mathbb{Z}} f\left(\frac{\pi m}{a}\right) J_{m, \lambda_{a}^{*}}(x)\right| \leq \sup _{x \in \mathbb{R}} \sum_{m \in \mathbb{Z}}\left|J_{m, \lambda_{a}^{*}}(x)\right| \\
=(2 a)^{-1} \sup _{x \in \mathbb{R}} \sum_{m \in \mathbb{Z}}\left|\left(\mathbf{F} \lambda_{a}^{*}\right)\left(x-\frac{\pi m}{a}\right)\right| \\
=2 \sup _{x \in \mathbb{R}} \sum_{m \in \mathbb{Z}}\left|\frac{\cos ((a x-\pi m) / 2)-\cos (a x-\pi m)}{(a x-\pi m)^{2}}\right| .
\end{array}
$$

Observe that the function

$$
\varphi(x):=\sum_{m \in \mathbb{Z}}\left|\frac{\cos ((a x-\pi m) / 2)-\cos (a x-\pi m)}{(a x-\pi m)^{2}}\right|
$$

is $\pi m / a$-periodic. Consequently, we can assume that $x \in[0, \pi / a]$ and

$$
\begin{aligned}
& 2 \sup _{x \in \mathbb{R}} \sum_{m \in \mathbb{Z}}\left|\frac{\cos ((a x-\pi m) / 2)-\cos (a x-\pi m)}{(a x-\pi m)^{2}}\right| \\
=2 & \sup _{x \in[0, \pi / a]} \sum_{m \in \mathbb{Z}}\left|\frac{\cos ((a x-\pi m) / 2)-\cos (a x-\pi m)}{(a x-\pi m)^{2}}\right| \\
= & 2\left(\sup _{x \in[0, \pi / a]} \sum_{|m| \geq 2}+\sup _{x \in[0, \pi / a]} \sum_{m=\{0,1,-1\}}\right)^{\cdot}
\end{aligned}
$$

Clearly,

$$
\begin{aligned}
& \sup _{x \in[0, \pi / a]} \sum_{m \leq-2} \leq 2 \pi^{-2} \sum_{m \geq 2} m^{-2}<2 \pi^{-2} \int_{1}^{\infty} m^{-2} d m<2 \pi^{-2}, \\
& \sup _{x \in[0, \pi / a]} \sum_{m \geq 2} \leq 2 \pi^{-2} \sum_{m \geq 1} m^{-2}<2 \pi^{-2}\left(1+\int_{1}^{\infty} m^{-2} d m\right)<4 \pi^{-2},
\end{aligned}
$$

and

$$
\sup _{x \in[0, \pi / a]} \sum_{m=\{0,1,-1\}}<3 \cdot \frac{3}{8}
$$

Hence,

$$
\left\|\mathbf{P}_{\lambda_{a}^{*}} \mid C(\mathbb{C}) \longrightarrow C(\mathbb{C})\right\|<2\left(6 \pi^{-2}+9 / 8\right)<3.5 .
$$


Consider the set $A_{\infty, \delta} U M$ of analytic in the strip $|\Im z| \leq \delta$ functions $f(z)$. Let $M:=\sup _{|\Im z| \leq \delta}|f(z)|$ and

$$
E\left(f, W_{\sigma}(\mathbb{R}), L_{\infty}(\mathbb{R})\right):=\inf _{g \in W_{\sigma}(\mathbb{R})}\|f-g\|_{L_{\infty}(\mathbb{R})} .
$$

be the best approximation of $f(z)$ by the space $W_{\sigma}(\mathbb{R})$ and

$$
E\left(A_{\infty, \delta} U M, W_{\sigma}(\mathbb{R}), L_{\infty}(\mathbb{R})\right):=\sup _{f \in A_{\infty, \delta} U M} \inf _{g \in W_{\sigma}(\mathbb{R})}\|f-g\|_{L_{\infty}(\mathbb{R})} .
$$

be the best approximation of the function class $A_{\infty, \delta} U M$ by $W_{\sigma}(\mathbb{R})$. It is known that for sufficiently large $\sigma$,

$$
E\left(A_{\infty, \delta} U M, W_{\sigma}(\mathbb{R}), L_{\infty}(\mathbb{R})\right) \leq \frac{4 M}{\pi} \sum_{k=0}^{\infty} \frac{(-1)^{k}}{(2 k+1) \cosh ((2 k+1) \sigma \delta)}
$$

(see, e.g., [22], p.320). Observe that

$$
\frac{4 M}{\pi} \sum_{k=0}^{\infty} \frac{(-1)^{k}}{(2 k+1) \cosh ((2 k+1) \sigma \delta)}<\frac{4 M}{\pi} \frac{1}{\cosh (\sigma \delta)}<\frac{8 M}{\pi} e^{-\sigma \delta} .
$$

Hence,

$$
E\left(A_{\infty, \delta} U M, W_{\sigma}(\mathbb{R}), L_{\infty}(\mathbb{R})\right)<\frac{8 M}{\pi} e^{-\sigma \delta} .
$$

The density function $p_{\tau}(y)$ can be approximated as

$$
\begin{aligned}
& p_{\tau}(y)=\frac{1}{2 \pi} e^{-\omega y} \int_{\mathbb{R}} e^{i y x-\tau \psi(x+i \omega)} d x=e^{-\omega y} \mathbf{F}^{-1}\left(e^{-\tau \psi(x+i \omega)}\right)(y) \\
\approx & \frac{1}{2 \sigma} e^{-\omega y} \sum_{m \in \mathbb{Z}} \exp \left(-\tau \psi\left(\frac{\pi m}{\sigma}+i \omega\right)\right) \mathbf{F}^{-1}\left(\mathbf{F} \lambda_{a}^{*}\right)\left(-x+\frac{\pi m}{\sigma}\right)(y) \\
= & \frac{1}{2 \sigma} e^{-\omega y} \lambda_{a}^{*}(y) \sum_{m \in \mathbb{Z}} \exp \left(-\tau \psi\left(\frac{\pi m}{\sigma}+i \omega\right)\right) \exp \left(\frac{i \pi m y}{\sigma}\right):=p_{\tau}^{*}(y, \sigma) .
\end{aligned}
$$

Hence for $F_{\text {call }}\left(S_{t}, t\right)$ we have the following approximation

$$
F_{\text {call }, \sigma}^{*}\left(S_{t}, t\right)=e^{-r \tau} \int_{-\infty}^{\infty}\left(e^{y}-K\right)_{+} p_{\tau}^{*}(y-x, \sigma) d y
$$

The next statement shows that $F_{\text {call, } \sigma}^{*}\left(S_{t}, t\right)$ converges exponentially fast to $F_{\text {call }}\left(S_{t}, t\right)$ as $\sigma \rightarrow \infty$. 
Theorem 11. Let $A>0$ be an absolute constant, $M(t):=\max \left\{e^{-t \psi(z+i \omega)} \mid\right.$ $z \in \mathbb{R}\}, \delta:=\min \left\{\lambda_{+}-1-\kappa, 1+\kappa-\lambda_{-}\right\}, \kappa>0, \omega=1+\kappa, \theta:=-(\max \{\nu, 1\})^{-1}$ and $\tau:=T-\tau$. If $\sigma \rightarrow \infty$ then

$$
\left|F_{\text {call }}\left(S_{t}, t\right)-F_{\text {call }, \sigma}^{*}\left(S_{t}, t\right)\right| \leq e^{-r \tau+\omega x} \frac{A\left(M(\tau)+\tau^{\theta}\right) K^{1-\omega}}{(\omega-1)}(\sigma \delta)^{1 / \nu} e^{-\sigma \delta} .
$$

Proof. Let $\tau:=T-t$ be the time to expiry and $e^{x}:=S_{t}$ then

$$
\begin{gathered}
F_{\text {call }}\left(S_{t}, t\right):=e^{-r \tau} \int_{\mathbb{R}} G(x+y) p_{\tau}(y) d y=e^{-r \tau} \int_{\mathbb{R}} G(y) p_{\tau}(y-x) d y \\
=e^{-r \tau} \int_{\mathbb{R}}\left(e^{y}-K\right)_{+} p_{\tau}(y-x) d y=e^{-r \tau} \int_{\ln K}\left(e^{y}-K\right) p_{\tau}(y-x) d y \\
=\frac{1}{2 \pi} e^{-r \tau} \int_{\ln K}\left(e^{y}-K\right)\left(\int_{\mathbb{R}} e^{i(y-x) \xi-\tau \psi(\xi)} d \xi\right) d y .
\end{gathered}
$$

Consider a regular Lévy process of exponential type $\left(\lambda_{-}, \lambda_{+}\right)$of order $\nu$. Its characteristic exponent (by the definition) admits analytic extension into the strip $\Im z \in\left[\omega_{-}, \omega_{+}\right] \subset\left(\lambda_{-}, \lambda_{+}\right)$and can be written in the form $\psi(\xi)=$ $-i \mu \xi+\phi(\xi),|\phi(\xi)| \asymp|\xi|^{\nu}$ as $\xi \rightarrow \infty, \Im \xi \in\left(\lambda_{-}, \lambda_{+}\right)$. Using Cauchy's theorem, changing variable $\xi=z+i \omega$ and simplifying we get

$$
\begin{gathered}
F_{\text {call }}\left(S_{t}, t\right)=\frac{1}{2 \pi} e^{-r \tau} \int_{\ln K}^{\infty}\left(e^{y}-K\right)\left(\int_{i \omega-\infty}^{i \omega+\infty} e^{i(y-x) \xi-\tau \psi(\xi)} d \xi\right) d y \\
=\frac{1}{2 \pi} e^{-r \tau} \int_{\ln K}^{\infty}\left(e^{y}-K\right)\left(\int_{\mathbb{R}} e^{i(y-x)(z+i \omega)-\tau \psi(z+i \omega)} d z\right) d y \\
=\frac{1}{2 \pi} e^{-r \tau} \int_{\ln K}^{\infty}\left(e^{(1-\omega) y}-K e^{-\omega y}\right)\left(\int_{\mathbb{R}} e^{i y z-i x z+\omega x-\tau \psi(z+i \omega)} d z\right) d y \\
=\frac{1}{2 \pi} e^{-r \tau+\omega x} \int_{\ln K}^{\infty}\left(e^{(1-\omega) y}-K e^{-\omega y}\right)\left(\int_{\mathbb{R}} e^{i(y-x) z-\tau \psi(z+i \omega)} d z\right) d y \\
=e^{-r \tau+\omega x} \int_{\ln K}^{\infty}\left(e^{(1-\omega) y}-K e^{-\omega y}\right)\left(\mathbf{F}^{-1}\left(e^{-\tau \psi(z+i \omega)}\right)\right)(y-x) d y
\end{gathered}
$$

for any $\omega>1, \lambda_{-}<\omega<\lambda_{+}$. Applying Theorem 10 and (7) we get that for any $g \in W_{\sigma}$,

$$
E(z, \tau, \sigma, \omega):=\left|e^{-\tau \psi(z+i \omega)}-\sum_{m \in \mathbb{Z}} \exp \left(-\tau \psi\left(\frac{\pi}{\sigma} m+i \omega\right)\right) J_{m, \lambda_{\sigma}^{*}}(z)\right|
$$




$$
\begin{gathered}
=\left|e^{-\tau \psi(z+i \omega)}-\sum_{m \in \mathbb{Z}} \exp \left(-\tau \psi\left(\frac{\pi}{\sigma} m+i \omega\right)\right) \frac{1}{2 \sigma} 2 \pi\left(\mathbf{F}^{-1} \lambda_{\sigma}^{*}\right)\left(z-\frac{\pi}{\sigma} m\right)\right| \\
=\mid e^{-\tau \psi(z+i \omega)}-g(z+i \omega)+g(z+i \omega) \\
-\sum_{m \in \mathbb{Z}} \exp \left(-\tau \psi\left(\frac{\pi}{\sigma} m+i \omega\right)\right) \frac{\pi}{\sigma}\left(\mathbf{F}^{-1} \lambda_{\sigma}^{*}\right)\left(z-\frac{\pi}{\sigma} m\right) \mid \\
=\mid e^{-\tau \psi(z+i \omega)}-g(z+i \omega) \\
-\sum_{m \in \mathbb{Z}}\left(\exp \left(-\tau \psi\left(\frac{\pi}{\sigma} m+i \omega\right)\right)-g\left(\frac{\pi}{\sigma} m+i \omega\right)\right) \frac{\pi}{\sigma}\left(\mathbf{F}^{-1} \lambda_{\sigma}^{*}\right)\left(z-\frac{\pi}{\sigma} m\right) \mid \\
\leq\left|e^{-\tau \psi(z+i \omega)}-g(z+i \omega)\right| \\
\sum_{m \in \mathbb{Z}}\left|\exp \left(-\tau \psi\left(\frac{\pi}{\sigma} m+i \omega\right)\right)-g\left(\frac{\pi}{\sigma} m+i \omega\right)\right|\left|\frac{\pi}{\sigma}\left(\mathbf{F}^{-1} \lambda_{\sigma}^{*}\right)\left(z-\frac{\pi}{\sigma} m\right)\right| \\
\leq E\left(A_{\infty, \delta} U M, W_{\sigma}(\mathbb{R}), L_{\infty}(\mathbb{R})\right)\left(1+\left\|\mathbf{P}_{\lambda_{\sigma}} \mid C(\mathbb{C}) \longrightarrow C(\mathbb{C})\right\|\right) \\
<\frac{8 M}{\pi}(1+3.5) e^{-\sigma \delta}<11.6 M e^{-\sigma \delta},
\end{gathered}
$$

where $M=M(\tau):=\max _{z \in \mathbb{R}} e^{-\tau \psi(z+i \omega)}$ and

$$
\delta:=\min \left\{\lambda_{+}-1-\kappa, 1+\kappa-\lambda_{-}\right\}, \kappa>0, \omega=1+\kappa .
$$

It means that

$$
\max _{z \in[-R, R]} E(z, \tau, \sigma, \omega)<11.6 M e^{-\sigma \delta}
$$

and

$$
\begin{gathered}
\max _{z \in(-\infty,-R] \cup[R, \infty)}\left|e^{-\tau \psi(z+i \omega)}-\sum_{m \in \mathbb{Z}} \exp \left(-\tau \psi\left(\frac{\pi}{\sigma} m+i \omega\right)\right) J_{m, \lambda_{\sigma}^{*}}\left(z-\frac{\pi}{\sigma} m\right)\right| \\
\leq\left(1+\left\|\mathbf{P}_{\lambda_{\sigma}^{*}} \mid C(\mathbb{C}) \longrightarrow C(\mathbb{C})\right\|\right) \\
\quad \times \max \left(\max _{z \in[R, \infty)}\left|e^{-\tau \psi(z+i \omega)}\right|, \max _{z \in(-\infty,-R]}\left|e^{-\tau \psi(z+i \omega) \mid}\right|\right) \leq 4.5 C_{\omega} e^{-\tau R^{\nu}},
\end{gathered}
$$

where $C_{\omega}$ depends just on $\omega$. Hence

$$
\left|\frac{1}{2 \pi} \int_{\mathbb{R}} e^{-i y z}\left(e^{-\tau \psi(z+i \omega)}-\sum_{m \in \mathbb{Z}} \exp \left(-\tau \psi\left(\frac{\pi}{\sigma} m+i \omega\right)\right) J_{m, \lambda_{\sigma}^{*}}\left(z-\frac{\pi}{\sigma} m\right)\right) d z\right|
$$




$$
\begin{gathered}
=\frac{1}{2 \pi} \mid\left(\int_{[-R, R]}+\int_{(-\infty,-R]}+\int_{[R, \infty)}\right) e^{-i y z} \\
\times\left(e^{-\tau \psi(z+i \omega)}-\sum_{m \in \mathbb{Z}} \exp \left(-\tau \psi\left(\frac{\pi}{\sigma} m+i \omega\right)\right) J_{m, \lambda_{\sigma}^{*}}\left(z-\frac{\pi}{\sigma} m\right)\right) d z \mid \\
\leq \frac{1}{2 \pi} \cdot 11.6 \cdot 2 R M(\tau) e^{-\sigma \delta}+\frac{1}{2 \pi} \cdot 2 \cdot 4.5 C_{\omega} \int_{[R, \infty)} e^{-\tau z^{\nu}} d z \\
<3.7 R M(\tau) e^{-\sigma \delta}+1.5 C_{\omega} \int_{[R, \infty)} e^{-\tau z^{\nu}} d z
\end{gathered}
$$

To estimate the last integral observe that if $\nu \geq 1$ then

$$
\begin{gathered}
\int_{[R, \infty)} e^{-\tau z^{\nu}} d z=\nu^{-1} \tau^{-\nu^{-1}} \int_{\left[\tau R^{\nu}, \infty\right)} e^{-z} z^{1 / \nu-1} d z \\
<\nu^{-1} \tau^{-\nu^{-1}} \int_{\left[\tau R^{\nu}, \infty\right)} e^{-z} d z=\nu^{-1} \tau^{-\nu^{-1}} e^{-\tau R^{\nu}}
\end{gathered}
$$

If $0<\nu<1$ then

$$
\begin{gathered}
\int_{\left[\tau R^{\nu}, \infty\right)} e^{-z} z^{1 / \nu-1} d z=e^{-\tau R^{\nu}}\left(\tau^{\nu^{-1}-1}\right) R^{1-\nu}+\frac{\nu}{1-\nu} \int_{\left[\tau R^{\nu}, \infty\right)} e^{-z} z^{1 / \nu-2} d z \\
<e^{-\tau R^{\nu}}\left(\tau^{\nu^{-1}-1}\right) R^{1-\nu}+\frac{\nu}{1-\nu} R^{-\nu} \int_{\left[\tau R^{\nu}, \infty\right)} e^{-z} z^{1 / \nu-1} d z
\end{gathered}
$$

or

$$
\int_{\left[\tau R^{\nu}, \infty\right)} e^{-z} z^{1 / \nu-1} d z<e^{-\tau R^{\nu}}\left(\tau^{\nu^{-1}-1}\right) R^{1-\nu}\left(1-\frac{\nu}{1-\nu} R^{-\nu}\right)^{-1}
$$

and

$$
\int_{[\tau R, \infty)} e^{-\tau z^{\nu}} d z<\nu^{-1} t^{-\nu^{-1}}\left(1-\frac{\nu}{1-\nu} R^{-\nu}\right)^{-1} e^{-\tau R^{\nu}}\left(\tau^{\nu^{-1}-1}\right) R^{1-\nu}
$$

i.e.,

$$
\begin{gathered}
I(R, \tau, \nu):=\int_{[R, \infty)} e^{-\tau z^{\nu} d z} \\
<\frac{e^{-\tau R^{\nu}\left(\tau^{\nu^{-1}-1}\right) R^{(1-\nu)_{+}}}}{\nu \tau^{\nu^{-1}}}\left\{\begin{array}{cc}
1, & \nu \geq 1, \\
\left(1-\frac{\nu R^{-\nu}}{1-\nu}\right)^{-1}, & 0<\nu<1 .
\end{array}\right.
\end{gathered}
$$


Finally, substituting (9) into (8) we get

$$
\begin{gathered}
d:=\mid \int_{\ln K}^{\infty}\left(e^{(1-\omega) y}-K e^{-\omega y}\right)\left(\mathbf{F}\left(e^{-\tau \psi(z+i \omega)}\right)\right)(y-x) d y \\
-\int_{\ln K}^{\infty}\left(e^{(1-\omega) y}-K e^{-\omega y}\right) \\
\left(\mathbf{F}\left(\sum_{m \in \mathbb{Z}} \exp \left(-\tau \psi\left(\frac{\pi}{\sigma} m+i \omega\right)\right) J_{m, \lambda_{\sigma}^{*}}\left(z-\frac{\pi}{\sigma} m\right)\right)\right)(y-x) d y \mid \\
<\left(3.7 R M(\tau) e^{-\sigma \delta}+1.5 I(R, \tau, \nu)\right) \int_{\ln K}^{\infty}\left(e^{(1-\omega) y}-K e^{-\omega y}\right) d y \\
<\left(3.7 R M(\tau) e^{-\sigma \delta}+1.5 I(R, \tau, \nu) C_{\omega}\right) \int_{\ln K}^{\infty} e^{(1-\omega) y} d y \\
=\left(3.7 R M(\tau) e^{-\sigma \delta}+1.5 I(R, \tau, \nu) C_{\omega}\right) \frac{1}{\omega-1} K^{1-\omega} .
\end{gathered}
$$

Put $R=(\sigma \delta)^{1 / \nu}$ then

$$
d \leq A\left(M(\tau)+\tau^{\theta}\right) K^{1-\omega}(\omega-1)^{-1}(\sigma \delta)^{1 / \nu} e^{-\sigma \delta}, \sigma \rightarrow \infty
$$

where $A$ is an absolute constant.

\section{References}

[1] N. I. Akhiezer, Lectures on approximation theory, Nayka, Moskow (1965).

[2] D. L. Applebaum, Lévy processes and Stochastic Calculus, Cambridge University Press, Cambridge (2009).

[3] O. E. Barndorff-Nielsen, Exponentially decreasing distributions for the logarithm of particle size, Proc. Roy. Soc. London, Ser. A 353 (1977), 401-419.

[4] J-P. Bouchaud, M. Potters, Theory of financial risk, Cambridge University press, Cambridge (2000).

[5] S. I. Boyarchenko, S. Z. Levendorskii, Non-gaussian Merton-Black-Scholes theory, World Scientific, Advanced Series on Statistical Science \& Applied Probability, Vol. 9 (2002). 
[6] I. I. Gihman, A. V. Skorohod, The Theory of Stochastic Processes I, Springer-Verlag (1974).

[7] I. I. Gihman, A. V. Skorohod, The Theory of Stochastic Processes II, Springer-Verlag (1975).

[8] I. I. Gihman, A. V. Skorohod, The Theory of Stochastic Processes III, Springer-Verlag (1979).

[9] E. F. Fama, The behaviour of stock market prices, Journ. of Business, 38 (1965), 34-105.

[10] I. Koponen, Analytic approach to the problem of convergence of truncated Lévy flights tovard the Gaussian stochastic process, Physics Review, 52 (1995), 1197-1199.

[11] S. Levendorskiı̌, J. Xie, Fast pricing and calculation of sensitivities of OTM Europian options under Lévy process (private communication).

[12] B. B. Mandelbrot, The variation of certain speculative prices, J. of Business, 36 (1963), 394-419.

[13] R. N. Mantegna, H. E. Stanley, Stochastic process with ultraslow convergence to a gaussian: the truncated Lévy flight, Phys. Rev. Lett., 73 (1994), 946-949.

[14] R. N. Mantegna, H. E. Stanley, An introduction to Econophysics. Correlation and complexity in Finance, Cambridge University Press, Cambridge (2000).

[15] H. P. McKean, Stochastic integrals, The Rockefeller University, Academic Press, New York (1969).

[16] E. A. Novikov, Infinitely divisible distributions in turbulence, Physics Review, 50 (1994), 3303-3305.

[17] K. Sato, Lévy Processes and Infinitely Divisible Distributions, Cambridge University Press (1999).

[18] C. E. Shannon, A Mathematical Theory of Communication, The Bell System Technical Journal, 27, 3 (1948), 379-423.

[19] C. E. Shannon, W. Weaver, A Mathematical Theory of Communication, Univ of Illinois Press (1949). 
[20] C. E. Shannon, Papers on information theory and cybernetics, Mir, Moscow (1963).

[21] V. M. Tikhomirov, Harmonics and splines as optimal tools for approximation and recovery, Russian Math. Surveys, 50 (1995), 125-174.

[22] A. F. Timan, Approximation theory of functions of real variable, Gos. Izd., Moskov (1960). 